\title{
SUBGROUPS OF FINITE GROUPS WITH A STRONG COVER-AVOIDANCE PROPERTY
}

\author{
A. BALLESTER-BOLINCHES, LUIS M. EZQUERRO ${ }^{\bowtie}$ and ALEXANDER N. SKIBA
}

\author{
(Received 9 September 2008)
}

\begin{abstract}
A subgroup $A$ of a group $G$ has the strong cover-avoidance property in $G$, or $A$ is a strong CAP-subgroup of $G$, if $A$ either covers or avoids every chief factor of every subgroup of $G$ containing $A$. The main aim of the present paper is to analyse the impact of the strong cover and avoidance property of the members of some relevant families of subgroups on the structure of a group.
\end{abstract}

2000 Mathematics subject classification: primary 20D10, 20D15, 20D20.

Keywords and phrases: finite group, cover-avoidance property, saturated formation.

\section{Introduction}

In this paper all groups are assumed to be finite. If a subgroup $A$ of a group $G$ has the property that either $H A=K A$ or $A \cap H=A \cap K$ for every chief factor $H / K$ of $G$, then $A$ is said to have the cover-avoidance property in $G$ and is called a $C A P$ subgroup of $G$. Over the past 35 years or so, the cover-avoidance property has attracted the attention of many authors. Some were interested in discovering distinguished families of $C A P$-subgroups, mainly in the soluble universe, while others discovered some characterizations of soluble and supersoluble groups, or their corresponding local versions, in terms of $C A P$-subgroups. For an overview, the reader may consult $[1, \mathrm{Ch} .4,2,7]$ and the works cited therein. The present article is a further contribution to this subject.

One notable feature of a supersoluble group which is generally absent from a nonsupersoluble one is that the cover-avoidance property is inherited in subgroups. This means that if $A$ is a $C A P$-subgroup of $G$ and $A$ is a subgroup of $B$, then $A$ is not, in general, a $C A P$-subgroup of $B$ (see [2, Example 3]). Therefore it is quite natural to inquire about the cover-avoidance property in subgroups. This motivates the following definition.

The research of the first author is supported by Proyecto MTM2007-68010-C03-02, Ministerio de Educación y Ciencia de España. The research of the second author is supported by Proyecto MTM200768010-C03-01, Ministerio de Educación y Ciencia de España.

(c) 2009 Australian Mathematical Society 0004-9727/2009 \$16.00 
Definition 1. Let $A$ be a subgroup of a group $G$. We say that $A$ is a strong $C A P$ subgroup of $G$ if $A$ is a $C A P$-subgroup of any subgroup of $G$ containing $A$.

Supersoluble groups can be thought of as those groups all of whose subgroups are strong $C A P$-subgroups. It is natural to ask whether one can impose this property on the subgroups of a more restricted family and not lose supersolubility. The answer to that question is contained in the following general result.

THEOREM A. Let $\mathfrak{F}$ be a saturated formation containing all supersoluble groups and $G$ a group with a normal subgroup $E$ such that $G / E \in \mathfrak{F}$. Suppose that every noncyclic Sylow subgroup $P$ of the generalized Fitting subgroup $F^{*}(E)$ of $E$ has a subgroup $D$ such that $1<|D|<|P|$ and all subgroups $H$ of $P$ with order $|H|=|D|$ and with order $2|D|$ (if $P$ is a nonabelian 2-group) are strong CAP-subgroups of $G$. Then $G \in \mathfrak{F}$.

For the saturated formation of all supersoluble groups we have the following result.

Corollary 2. A group $G$ is supersoluble if and only if every noncyclic Sylow subgroup $P$ of $F^{*}(G)$ has a subgroup $D$ such that $1<|D|<|P|$ and all subgroups $H$ of $P$ with order $|H|=|D|$ and with order $2|D|$ (if $P$ is a nonabelian 2-group) are strong CAP-subgroups of $G$.

\section{Preliminary results}

We begin with some elementary properties of the strong $C A P$-subgroups which will be extremely useful in what follows.

LEMMA 3. Let $G$ be a group and $N$ a normal subgroup of $G$.

(1) If $H$ is a CAP-subgroup (strong CAP-subgroup) of $G$, then so is $H N$.

(2) If $A$ is subgroup of $G$, then $A N / N$ is a CAP-subgroup (strong CAP-subgroup) of $G / N$ if and only if $A$ is a CAP-subgroup (strong CAP-subgroup) of $G$.

ProOF. See [5, Lemma 1].

Let $H / K$ be a chief factor of a group $G$. The innerizer of $H / K$ is defined to be the subgroup $C_{G}^{*}(H / K)=H C_{G}(H / K)$. Note that if $H / K$ is abelian, then $C_{G}^{*}(H / K)=C_{G}(H / K)($ see [1, Definition 1.2.2]) .

A group $G$ is said to be quasinilpotent if $G$ is the common innerizer of every chief factor of $G$. The product of two normal quasinilpotent subgroups of a group $G$ is quasinilpotent and so each group $G$ has a unique normal subgroup which is maximal subject to being quasinilpotent. This subgroup is called the generalized Fitting subgroup of $G$ and denoted by $F^{*}(G)$. It is clear that $F^{*}(G)$ is a characteristic subgroup of $G$ which coincides with $F(G)$ if it is soluble. The reader may consult $[9, \mathrm{Ch} . \mathrm{X}]$ for further properties of quasinilpotent groups and the generalized Fitting subgroup.

The class of all quasinilpotent groups is a solubly saturated formation (see $[4$, p. 579]). Therefore we have the following result. 
LEMMA 4. Let $p$ be a prime and $P$ a normal p-subgroup of a group $G$. Then $F^{*}(G / \Phi(P))=F^{*}(G) / \Phi(P)$. If $P$ is contained in $Z(G)$, then $F^{*}(G / P)=$ $F^{*}(G) / P$.

For a group $G$ and a subgroup $D$ of $G$, the family of all subgroups $H$ of $G$ such that $|H|=|D|$ is denoted by $\Theta_{D}(G)$. If $G$ is a 2-group, we shall assume that $\Theta_{D}(G)$ also includes the subgroups $X$ of $G$ such that $|X|=2|D|$.

We often require the next lemma in our proofs. It allows us to invoke Lemma 3 at the appropriate point.

Lemma 5. Let $p$ be a prime and let $P$ be a p-subgroup of a group $G$ containing $a$ noncyclic minimal normal subgroup $N$ of $G$. Assume that $P$ has a nontrivial proper subgroup $D$ such that every subgroup in $\Theta_{D}(P)$ is a CAP-subgroup (strong CAPsubgroup) of $G$. Then $P / N$ has a subgroup with the same property in $G / N$.

Proof. If $|D|$ were less than $|N|$, then $N$ would have a subgroup in $\Theta_{D}(P)$ which neither covers nor avoids $N$. Hence $|N| \leq|D|$. Assume that $|N|=|D|$. If $\left|\Theta_{D}(P)\right|>1$, then $\Theta_{D}(P)$ has subgroups avoiding $N$. Let $F$ be one of them and let $Z$ be a minimal normal subgroup of $F N$ contained in $N$. If $L$ is a maximal subgroup of $F$, it follows that $Z L \in \Theta_{D}(P)$. Then $N$ is contained in $Z L$. This implies that $Z=N$ and $N$ is cyclic, contrary to our assumption. Therefore we have $\Theta_{D}(P)=\{N\}$. Since $|N|>p, P$ has to be cyclic by [8, Kapitel III, Satz 8.3]. This contradiction leads to $|N|<|D|$. In this case $P$ has a subgroup $E$ in $\Theta_{D}(P)$ containing $N$ and so the nonempty family of subgroups $\Theta_{E / N}(P / N)$ of $P / N$ is composed of $C A P$-subgroups (strong $C A P$-subgroups) of $G / N$.

We now obtain one of the main results of this section.

THEOREM 6. Let $p$ be the smallest prime dividing the order of a soluble group $G$. Assume that every Sylow p-subgroup $P$ of $G$ has a nontrivial proper subgroup $D$ such that every subgroup in $\Theta_{D}(P)$ is a strong CAP-subgroup of $G$. Then $G$ is p-nilpotent.

PROOF. Let $P$ be a Sylow $p$-subgroup of $G$ and let $D$ be a nontrivial proper subgroup of $P$ such that every member of $\Theta_{D}(P)$ is a strong $C A P$-subgroup of $G$. We proceed by induction on $|G|$.

Suppose that $O_{p^{\prime}}(G) \neq 1$ and let $N$ be a minimal normal $q$-subgroup of $G$ for some prime $q$ such that $p \neq q$. Then $G / N$ fulfils the hypothesis and so $G / N$ is $p$-nilpotent by induction. Then $G$ is $p$-nilpotent, as required. Thus we can assume $O_{p^{\prime}}(G)=1$.

Suppose that $|D|>p$. Let $N$ be a minimal normal subgroup of $G$. Then $N$ is contained in $P$. If $N$ is not cyclic, the hypotheses of Lemma 5 hold for the group $G / N$. By induction we have that $G / N$ is $p$-nilpotent. Assume that $N$ is cyclic. Then $|N|=p$. Since $|D|>p$, then $P$ has a subgroup $E$ in $\Theta_{D}(P)$ containing $N$ and so the nonempty family of subgroups $\Theta_{E / N}(P / N)$ of $P / N$ is composed of strong $C A P$-subgroups of $G / N$. By induction, $G / N$ is $p$-nilpotent. Consequently we may assume that $G / N$ is $p$-nilpotent for every minimal normal subgroup $N$ of $G$. Since the class of all $p$-nilpotent groups is a saturated formation, we can assume that $G$ is a 
primitive group, that is, $G$ has a self-centralizing minimal normal subgroup $N$ which is complemented in $G$ by a core-free maximal subgroup of $G$. Since $O_{p^{\prime}}(G)=1$, it follows that $N$ is a $p$-group. If $N$ is cyclic, then $N$ is central in $P$. Then $P \leq C_{G}(N)=N$. But this is not possible. Therefore we may assume that $|N|>p$. Suppose that every subgroup in $\Theta_{D}(P)$ covers $N$. Then $N$ is contained in $\Phi(P)$. This contradicts the fact that $N$ is complemented in $G$. Hence there exists a subgroup $H \in \Theta_{D}(P)$ avoiding $N$. Consider a minimal normal subgroup $X$ of $N H$ and a maximal subgroup $J$ of $H$. Then $X$ is of order $p$ and so $X J$ is an element of $\Theta_{D}(P)$ which neither covers nor avoids $N$. This contradiction leads us to $|D|=p$.

Assume that there exists a minimal non- $p$-nilpotent subgroup $K$ of $G$. According to [8, Kapitel IV, Satz 5.4], $K$ has a normal Sylow $p$-subgroup $A$ such that $A$ has exponent $p$ if $p$ is odd and exponent at most 4 if $p=2$. Moreover, $A / \Phi(A)$ is a chief factor of $K$. The assumptions on $G$ imply that every subgroup of $A$ either covers or avoids $A / \Phi(A)$ and so $A$ is cyclic. Applying [8, Kapitel IV, Satz 2.8], $K$ is $p$ nilpotent. This contradiction shows that the group $G$ is $p$-nilpotent. The proof of the theorem is complete.

We shall naturally be interested in the study of CAP-subgroups of some characteristic subgroups associated with saturated formations. In this context, the following results turn out to be useful.

COROLLARY 7. Let $\mathfrak{F}$ be a saturated formation containing all supersoluble groups and $G$ a group with a soluble normal subgroup $E$ such that $G / E \in \mathfrak{F}$. Suppose that every noncyclic Sylow subgroup $P$ of $E$ has a nontrivial proper subgroup $D$ such that every subgroup in $\Theta_{D}(P)$ is a strong $C A P$-subgroup of $G$. Then $G \in \mathfrak{F}$.

PROOF. First we notice that if every Sylow subgroup of $E$ is cyclic, then the result holds. In that case, every chief factor $H / K$ of $G$ below $E$ is a cyclic group of order $q$ for some prime $q$. It follows that $G / C_{G}(H / K)$ is abelian of exponent dividing $q-1$. It implies that every chief factor of $G$ below $E$ is $\mathfrak{F}$-central in $G$ and then $G \in \mathfrak{F}$ by [4, Ch. IV, Theorem 5.7].

Assume that the result is false and let $G$ be a counterexample with $|G|+|E|$ minimal. Then $E \neq 1$. Let $p$ be the smallest prime dividing $|E|$. Then $E$ is $p$-nilpotent by [8, Kapitel IV, Satz 2.8] and Theorem 6. Let $A$ be a normal Hall $p^{\prime}$-subgroup of $E$. Then $A$ is normal in $G$. Suppose $A \neq 1$. Since the pair $(G / A, E / A)$ satisfies the hypothesis of the corollary, it follows that $G / A \in \mathfrak{F}$, and so $(G, A)$ fulfils the conditions of the corollary. The minimality of $(G, E)$ yields $G \in \mathfrak{F}$. This contradicts our initial assertion. Therefore $E$ is a $p$-group. By the previous remark, we can assume that $E$ is not cyclic and so it has a nontrivial proper subgroup $D$ such that every subgroup in $\Theta_{D}(E)$ is a strong $C A P$-subgroup of $G$.

Let $N$ be a minimal normal subgroup of $G$ contained in $E$. If $N$ is not cyclic, we apply Lemma 5 and the minimal choice of $G$ to conclude that $G / N \in \mathfrak{F}$. Assume that $|N|=p$. Suppose that $|D|=p$. Since $G \notin \mathfrak{F}$, there exists a maximal subgroup $M$ 
of $G$ such that $G=E M$. It is clear that $(M, E \cap M)$ satisfies the hypotheses of the corollary. Hence $M \in \mathfrak{F}$. Let $L$ be the $\mathfrak{F}$-residual of $G$. Applying [3, Proposition 1], $L / \Phi(L)$ is a chief factor of $G$ and the exponent of $L$ is $p$ if $p$ is odd and at most 4 if $p=2$. The assumption on $E$ implies that every subgroup of $L$ covers or avoids $L / \Phi(L)$. In particular, $L / \Phi(L)$ is cyclic and so it should be $\mathfrak{F}$-central. This is not possible. Hence, $|D|>p$. Then the pair $(G / N, E / N)$ satisfies the hypothesis of the corollary. By minimality of $G$, it follows that $G / N \in \mathfrak{F}$. Thus, $G / N \in \mathfrak{F}$ for every minimal normal subgroup $N$ of $G$ contained in $E$.

Let $A$ be a minimal normal subgroup of $G$ such that $A$ is not contained in $E$. Then $(G / A, E A / A)$ satisfies the hypotheses of the corollary. The minimal choice of $G$ yields $G / A \in \mathfrak{F}$. Hence $G \in \mathfrak{F}$, contrary to supposition. Consequently every minimal normal subgroup of $G$ is contained in $E$. Since $\mathfrak{F}$ is a saturated formation, it follows that $G$ has a unique minimal normal subgroup $N, G / N \in \mathfrak{F}$ and $N=O_{p}(G)$. In particular $N=E$ and so every subgroup in $\Theta_{D}(N)$ covers or avoids $N$. This final contradiction proves the corollary.

LEMMA 8. A quasinilpotent group $G$ is nilpotent if and only if every noncyclic Sylow subgroup $P$ of $G$ has a nontrivial proper subgroup $D$ such that all subgroups in $\Theta_{D}(P)$ are strong $C A P$-subgroups of $G$.

PROOF. Only the sufficiency of the condition is in doubt. Suppose that this is false and let $G$ be a minimal counterexample. Hence $G$ is not soluble. In particular, by the Feit-Thompson odd order theorem, $G$ is not 2-nilpotent and then $G$ has no cyclic Sylow 2-subgroups by [8, Kapitel IV, Satz 2.8]. Since $G$ is quasinilpotent, $G$ has a normal subgroup $R$ such that $G / R$ is a nonabelian simple group.

Let $\left\{p_{1}, p_{2}, \ldots, p_{t}\right\}$ be the set of all primes dividing $|G|$ such that a Sylow $p_{i}$-subgroup $P_{i}$ of $G$ is not cyclic. We may assume that $2=p_{1}$. Then $P_{i}$ has a nontrivial proper subgroup $D_{i}$ such that all subgroups in $\Theta_{D_{i}}\left(P_{i}\right)$ are strong $C A P$ subgroups of $G$. Suppose that for some $i$ there exists $H \in \Theta_{D_{i}}\left(P_{i}\right)$ such that $H \nsubseteq R$. Then $H$ does not avoid the chief factor $G / R$. Hence $G=H R$ and so $G / R$ is a $p_{i}$-group. This is a contradiction. Therefore we have $H \leq R$, for every subgroup $H \in \Theta_{D_{i}}\left(P_{i}\right), 1 \leq i \leq t$. In particular, 2 divides $|R|$. It follows that the hypothesis is still true for the $R$. Since $R$ is quasinilpotent, $R$ is nilpotent by the choice of $G$. Hence every chief factor of $G$ below $R$ is central in $G$, by [9, Ch. X, Corollary 13.7 Part(c)]. Let $Z$ be a minimal normal subgroup of $G$ contained in the Sylow 2-subgroup of $R$. Since $G$ is not 2-nilpotent, $G$ has a minimal non2-nilpotent subgroup $K$. According to [8, Kapitel IV, Satz 5.4], $K$ has a normal Sylow 2-subgroup $A$ such that $A$ has exponent at most 4. Moreover, $A / \Phi(A)$ is a chief factor of $K$. If $\left|D_{1}\right|=2$, then every subgroup $\Theta_{D_{1}}\left(P_{1}\right)$ would cover or avoid $A / \Phi(A)$. This would imply that $A$ is cyclic and so $K$ would be 2 -nilpotent by [8, Kapitel IV, Satz 2.8]. Hence $\left|D_{1}\right|>2$, so that the hypothesis holds for $G / Z$. Hence $G / Z$ is nilpotent by the choice of $G$. This means that $G$ is nilpotent. This contradiction completes the proof of the lemma. 


\section{The proof of Theorem A}

Assume, arguing by contradiction, that the theorem is not true and choose for $G$ a counterexample with $|G|+|E|$ minimal. Then $G$ has the following properties.

(1) $F=F^{*}(E)=F(E) \neq E$.

By Lemma $8, F^{*}(E)$ is nilpotent. Hence $F^{*}(E)=F(E)$. If $F^{*}(E)=E$, we can apply Corollary 7 to conclude that $G \in \mathfrak{F}$, which contradicts our choice of $G$. Hence $F^{*}(E)$ is a proper subgroup of $E$.

(2) Let $p$ the smallest prime dividing the order of $F$ and let $P$ be a Sylow $p$-subgroup of $F$. Write $V / P=F^{*}(E / P)$. Then $p>2$.

Suppose that $p=2$. Let $Q$ be a Sylow $q$-subgroup of $E$ for a prime $q \neq 2$. Then $P Q$ is 2-nilpotent by Corollary 7 (note that the class of all 2-nilpotent groups is a saturated formation containing all supersoluble groups). Since $P$ is a normal in $G$, it follows that $P Q$ is nilpotent. This happens for every odd prime $q$ and therefore $O^{2}(E) \leq C_{G}(P)$. Consider the normal subgroup $W=O^{2}(V) P$ of $E$. Then $W / P=O^{2}(V / P)$ is a quasinilpotent group and every chief factor of $W$ below $P$ is central in $W$. Hence $W$ is quasinilpotent and then $W \leq F^{*}(E)=F$. Thus, $W$ is nilpotent and $V / F$ is a 2-group. Therefore $V$ is soluble. This implies that $V / P$ is nilpotent. If $R$ is a Sylow 2-subgroup of $V$, then $R$ is normal in $V$. Let $H$ be a Hall $2^{\prime}$-subgroup of $V$. Since $H$ stabilizes the series $R \geq P \geq 1$, then $H \leq C_{V}(R)$, by [4, Ch. A, Corollary 12.4]. Hence $V$ is nilpotent and therefore $V=F$. The pair $(G / P, E / P)$ satisfies the hypotheses of the theorem. The minimal choice of $(G, E)$ implies that $G / P \in \mathfrak{F}$. Applying Corollary 7, $G \in \mathfrak{F}$, contrary to supposition. Hence $p$ is an odd prime.

(3) Let $X$ be a normal subgroup of $G$ contained in $P$. Suppose that $1=X_{0} \leq X_{1}$ $\leq \cdots \leq X_{t}=X$ is part of a chief series of $G$ below $X$. If $X_{i} / X_{i-1}$ is cyclic for all $i \in\{1, \ldots, t\}$, then $E$ centralizes $X_{i} / X_{i-1}$ for all $i$.

Let $C_{i}=C_{G}\left(X_{i} / X_{i-1}\right), 1 \leq i \leq t$. Since $G / C_{i}$ is cyclic, it follows that $G / C_{i} \in \mathfrak{F}$, $1 \leq i \leq t$. Let $C=C_{1} \cap C_{2} \cap \cdots \cap C_{t}$. Since $\mathfrak{F}$ is a residually closed, it follows that $G / C \in \mathfrak{F}$. Hence $G / E \cap C \in \mathfrak{F}$. Note that $F$ is contained in $E \cap C$. Therefore the pair $(G, E \cap C)$ satisfies the hypotheses of the theorem. The minimal choice of the pair $(G, E)$ implies that $E=E \cap C$.

(4) $P$ is not cyclic.

Suppose that $P$ is cyclic. Then every chief factor of $G$ below $P$ is cyclic and so $P$ fulfils the condition of step (3). Therefore $E$ stabilizes a chain of subgroups of $P$. By [4, Ch. A, Corollary 12.4], $E / C_{E}(P)$ is a $p$-group or, equivalently, $O^{p}(E) \leq C_{E}(P)$. Now we can argue as in step (2), replacing the prime 2 by $p$, and we deduce that $V / P$ is nilpotent. Since every chief factor of $V$ below $P$ is central in $V$, we deduce that $V$ is nilpotent. Therefore $V / P=F / P$. Now the pair $(G / P, E / P)$ satisfies the hypotheses of the theorem. By minimality of $(G, E)$, we have that $G / P \in \mathfrak{F}$. Applying Corollary $7, G \in \mathfrak{F}$, which contradicts our choice of $G$. 
(5) Let $D$ be a nontrivial proper subgroup $P$ such that all subgroups in $\Theta_{D}(P)$ are strong CAP-subgroups of $G$. Then $|D|>p$.

Suppose that $|D|=p$. Since $p$ is odd, $\Omega_{1}(P)$ possesses a characteristic subgroup $W$ of class at most 2 and of exponent $p$ such that every nontrivial $p^{\prime}$-automorphism of $\Omega_{1}(P)$ induces a nontrivial automorphism of $W$ (see [6, Ch. 5, Theorem 3.13]). It is clear that the assumptions on $G$ imply that every chief factor of $G$ below $W$ is cyclic. Applying step (3), $E$ stabilizes a chain of subgroups of $P$. By [4, Ch. A, Corollary 12.4], $E / C_{E}(W)$ is a $p$-group.

Consider an element $x \in E$ such that $x C_{E}(P)$ is a nontrivial $p^{\prime}$-element of $E / C_{E}(P)$. Let $\alpha_{x}$ denote the automorphism of $P$ induced by the conjugacy by $x$. Suppose that $\alpha_{x}$ acts nontrivially on $\Omega_{1}(P)$. Then $\alpha_{x}$ acts nontrivially on $W$. This is to say that $x C_{E}(W)$ is a nontrivial $p^{\prime}$-element of $E / C_{E}(W)$. Since $E / C_{E}(W)$ is a $p$-group, this is not possible. Hence, $\alpha_{x}$ acts trivially on $\Omega_{1}(P)$. By [6, Ch. 5, Theorem 3.10], this implies that $\alpha_{x}=1$, that is, $x \in C_{E}(P)$. This is to say that there is no nontrivial $p^{\prime}$-element of $E / C_{E}(P)$ or, in other words, that $E / C_{E}(P)$ is a $p$-group.

Then we can argue as in step (2) or step (4) to conclude that $G \in \mathfrak{F}$. This contradiction shows that $|D|>p$.

(6) $\Phi(P)=1$.

Assume that $\Phi(P) \neq 1$ and let $L$ be a minimal normal subgroup of $G$ contained in $\Phi(P)$. By Lemma $4, F^{*}(E / L)=F^{*}(E) / L$. For primes $q \neq p$, the noncyclic Sylow $q$-subgroups of $F^{*}(E) / L$ satisfy the hypotheses of the theorem by Lemma 3. If $P / L$ were cyclic, then $P / \Phi(P)$ would be cyclic and so would be $P$, against step (4). Hence, $P / L$ is noncyclic. If $D L / L$ is trivial, then $D=L$. By step (5) and [8, Kapitel III, Satz 8.2], there exists some $D_{0} \in \Theta_{D}(P)$ with $D_{0} \neq D$. Hence, in any case, the Sylow $p$-subgroups of $F^{*}(E) / L$ satisfy the hypotheses of the theorem, by Lemma 3. Hence $G / L \in \mathfrak{F}$ by minimality of $G$. Since $\Phi(P)$ is contained in $\Phi(G)$ and $\mathfrak{F}$ is saturated, it follows that $G \in \mathfrak{F}$, contrary to our supposition.

\section{(7) Conclusion.}

Let $N$ be a minimal normal subgroup of $G$ contained in $P$. Clearly $N$ is a proper subgroup of $P$. Since $\Phi(P)=1, P$ is a completely reducible $G$-module over GF $(p)$, the finite field of $p$-elements. Hence $P=N \times M$ for some normal subgroup $M \neq 1$ of $G$. Suppose that $|M|<|D|$. Then $P$ has a subgroup $H$ such that $M$ is contained in $H$ and $|H|=|D|$. This is not possible because $H$ neither covers nor avoids $N$. Hence $|D| \leq|M|$. In particular, $|P|>p^{2}$. Let $K$ be a nontrivial subgroup of $M$ such that $|K|=|D| / p$ (note that $|D|>p$ by step (5)) and let $Z$ be a minimal normal subgroup of $P$ contained in $N$. Then $Z$ has order $p$ and $Z K$ is a subgroup of $P$ of order $|D|$ which does not avoid $N$. Hence $Z=N$. By step (3), $E$ centralizes $N$ and then $F^{*}(E / N)=F^{*}(E) / N$. Since $P / N$ is noncyclic, we can argue as in step (6) to conclude that the noncyclic Sylow subgroups of $F^{*}(E / N)$ satisfy the hypotheses of the theorem. Hence $G / N \in \mathfrak{F}$ by minimality of $G$. Since $N$ is cyclic, it follows that $N$ is $\mathfrak{F}$-central in $G$. Hence $G \in \mathfrak{F}$. This final contradiction proves the theorem. 


\section{Acknowledgement}

Part of this research was carried out during a visit of the first author to the Department of Mathematics of the Australian National University, Canberra, Australia, in July and August 2008. He is grateful to the Department of Mathematics for its warm hospitality and financial support.

\section{References}

[1] A. Ballester-Bolinches and L. M. Ezquerro, Classes of Finite Groups (Springer, Dordrecht, 2006).

[2] A. Ballester-Bolinches, L. M. Ezquerro and A. N. Skiba, On second maximal subgroups of Sylow subgroups of finite groups, submitted.

[3] A. Ballester-Bolinches and M. C. Pedraza-Aguilera, 'On minimal subgroups of finite groups', Acta Math. Hungar. 73(4) (1996), 335-342.

[4] K. Doerk and T. Hawkes, Finite Soluble Groups (Walter de Gruyter, Berlin, 1992).

[5] L. M. Ezquerro, 'A contribution to the theory of finite supersolvable groups', Rend. Sem. Mat. Univ. Padova 89 (1993), 161-170.

[6] D. Gorenstein, Finite Groups (Harper \& Row, New York, 1968).

[7] X. Guo and K. P. Shum, 'Cover-avoidance properties and the structure of finite groups', J. Pure Appl. Algebra 181 (2003), 297-308.

[8] B. Huppert, Endliche Gruppen I, Grundlehren der Mathematischen Wissenschaften, 134 (Springer, Berlin, 1967).

[9] B. Huppert and N. Blackburn, Finite Groups III, Grundlehren der Mathematischen Wissenschaften, 243 (Springer, Berlin, 1982).

A. BALLESTER-BOLINCHES, Departament d'Àlgebra, Universitat de València, Dr. Moliner, 50, E-46100 Burjassot, València, Spain e-mail: Adolfo.Ballester@uv.es

LUIS M. EZQUERRO, Departamento of Matemáticas, Universidad Pública de Navarra, Campus de Arrosadía, E-31006 Pamplona, Navarra, Spain

e-mail: ezquerro@unavarra.es

ALEXANDER N. SKIBA, Department of Mathematics, Gomel State University F. Skorina, Gomel 246019, Belarus

e-mail: alexander.skiba49@gmail.com 